\title{
Gamma rays from gravitationally boosted neutralinos at the galactic center
}

\author{
Mirco Cannoni ${ }^{* \dagger}$ \\ Departamento de Física Aplicada, Facultad de Ciencias Experimentales, Universidad de \\ Huelva, 21071 Huelva, Spain \\ E-mail: mirco.cannoniedfa.uhu.es
}

\begin{abstract}
Neutralinos may be gravitationally boosted near the super-massive black hole at the galactic center so that they can have enough collision energy to annihilate into a stau pair. Since in some phenomenologically favored supersymmetric models the mass splitting between the neutralino and the lightest stau is a few GeVs, this channel may be allowed. In addition, staus can only decay into a tau lepton and another neutralino. We discuss the mechanisms behind this phenomenon and the gamma-ray spectrum and flux generated by the tau pair.
\end{abstract}

VIII International Workshop on the Dark Side of the Universe,

June 10-15, 2012

Rio de Janeiro, Brazil

* Speaker.

${ }^{\dagger}$ MultiDark fellow. 


\section{Introduction}

In one the favored regions of the parameter space of the constrained minimal supersymmetric standard model (CMSSM) where the lightest neutralino has the correct dark matter relic density, the lightest stau, $\tilde{\tau}_{1}$, is almost degenerate in mass with the neutralino [1]. This region is not characteristic of the CMSSM only, but also of the general MSSM without unification conditions. In fact the recent evidence at LHC of the Higgs boson with mass around $125 \mathrm{GeV}$ [2] and a decay width into two photons larger the in the Standard Model, single out a region of the MSSM parameter space where the neutralino and $\tilde{\tau}_{1}$ are light and degenerate [3].

The gravitational potential in the galactic center $(\mathrm{GC})$ is dominated by a super-massive black hole (BH) with mass $M_{\mathrm{BH}}=4 \times 10^{6} M_{\odot}$ and Schwarzschild radius $R_{\mathrm{S}}=2 G M_{\mathrm{BH}} / c^{2}=4 \times 10^{-7}$ pc. It has been suggested in [4] that particles captured by the $\mathrm{BH}$ can originate very high energy collision in the center of mass frame (CMF). In principle, hence, new annihilation channels into heavier states, kinematically forbidden for non relativistic particles, could be accessible.

We briefly discuss here the mechanism proposed in Ref. [5]: if dark matter is formed by neutralinos with the above properties, a new dominant annihilation channel is opened already for sub-relativistic neutralinos [6] not captured by the BH.

\subsection{Dark matter density profile at the GC}

The dark matter density distribution in the sub-parsec region at the GC cannot be resolved by present simulations nor by rotation curves measurement but must be inferred by physical considerations. The adiabatic growth of the $\mathrm{BH}$ at the center of the halo causes a steepening, called spike, of the initial halo profile toward the GC [7]. Adding scattering of DM particles off stars, capture by the $\mathrm{BH}$, self-annihilation and capture within stars during the evolution of the $\mathrm{DM}$ distribution, results in a profile, $\rho_{\mathrm{sp}}(r) \propto r^{-3 / 2}$ [8-12]. At a certain distance from the GC the density reaches a value such that self-annihilation itself acts to stop further rising reaching a constant value, commonly called annihilation plateau or core. In [12], however, it is argued that in the innermost region the density behave as $\rho_{\mathrm{ms}}(r) \propto r^{-1 / 2}$, a mild spike (MS). To this picture the adiabatic compression of the gravitational potential caused by the baryons already in the bulge of the galaxy [13] should also be added.

We hence model the profile considering that at the radius $r_{\mathrm{sp}} \approx 0.2 r_{\mathrm{h}}$, with $r_{\mathrm{h}}=1.67 \mathrm{pc}$ the influence radius of the $\mathrm{BH}, \mathrm{DM}$ density is given by a compressed Einasto profile, $\rho_{\mathrm{sp}}=5 \times 10^{6}$ $\mathrm{GeV} / \mathrm{cm}^{3}$ as in [11]. From here the profile is given by $\rho(r)=\rho_{\mathrm{sp}}\left(r / r_{\mathrm{sp}}\right)^{-\gamma_{\mathrm{sp}}}, \gamma_{\mathrm{sp}}=3 / 2$, up to the radius $r_{\mathrm{a}}$ where the density reaches the value $\rho_{\mathrm{a}}=m_{\chi} /(\sigma v)_{0} t_{f} .(\sigma v)_{0}$ is the annihilation cross section and $t_{f}=10 \mathrm{Gyr}[11,8]$ is the elapsed time since the formation of the spike. Finally, the inner MS is $\rho_{\mathrm{a}}\left(r / r_{\mathrm{a}}\right)^{-\gamma_{\mathrm{a}}}, \gamma_{\mathrm{a}}=1 / 2$, up to the limit $4 R_{\mathrm{S}}$. The radius $r_{\mathrm{a}}$ is found by matching the two power-laws, $r_{\mathrm{a}}=r_{\mathrm{sp}}\left(\rho_{\mathrm{a}} / \rho_{\mathrm{sp}}\right)^{-1 / \gamma_{\mathrm{sp}}}$.

Given a density described a power-law distribution, from the Jeans equation, the root mean squared velocity is $v(r) \approx\left(G M_{\mathrm{BH}} / r\right)^{1 / 2}[14,9,10]$, or in terms of the Schwarzschild radius, $v(r) / c \approx\left(R_{\mathrm{S}} / 2 r\right)^{1 / 2}$. Since a Keplerian orbit with $L_{c}$ would cross the horizon if the pericenter distance is less than $r_{\min }=4 R_{\mathrm{S}}$, hence we will consider safely $r>4 R_{\mathrm{S}}$. In this way, for example, at $r=10 R_{\mathrm{S}}$ we have $v / c \simeq 1 / \sqrt{20} \simeq 0.22$ and sub-relativistic velocities are possible. 


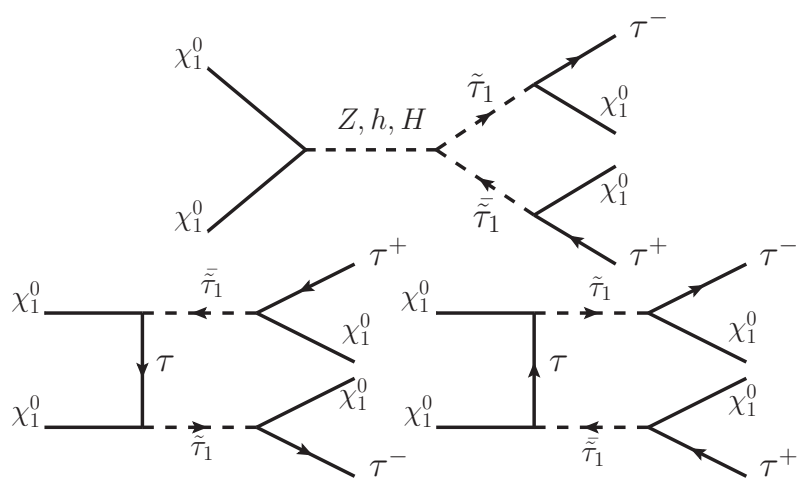

Figure 1: Diagrams for stau pair production and decay in neutralino annihilation.

\section{Large annihilation cross section into a stau pair}

The relic density constraint implies that the relative mass splitting with the $\tilde{\tau}_{1}, \delta=\left(m_{\tilde{\tau}}-\right.$ $\left.m_{\chi}\right) / m_{\chi}$, is typically less than $5 \%$. In the CMF the energy threshold for stau pair production is $\sqrt{s}=2 E_{\chi} \geq 2 m_{\tilde{\tau}}$, that implies $v / c \geq\left[1-1 /(1+\delta)^{2}\right]^{1 / 2}$. With $\delta=2 \%, v / c \geq 0.197$ : there exists thus a range of radii where the kinetic energy is high enough to reach the threshold of the process $\chi \chi \rightarrow \tilde{\tau}^{-} \tilde{\tau}^{+}$.

In Fig. 2, we show the relevant cross sections as a function of $\sqrt{s}$. The CMSSM points A and B, see Ref. [5] for details, predict a Higgs mass around 119-122 GeV and are compatible with a Higgs of mass $125 \mathrm{GeV}$ within the theoretical and the experimental errors. The cross section for stau pair-production near the threshold clearly dominates by one or two orders of magnitude the cross sections for annihilation. In the right panels we show the annihilation cross section times the particle relative velocity, as a function the CMF velocity of colliding neutralinos. Note that $\sigma v_{\text {rel }}$ near the threshold is at least an order of magnitude bigger than the freeze-out value $3 \times 10^{-26}$ $\mathrm{cm}^{3} / \mathrm{s}$. These values corresponds to $v / c \sim 0.1-0.2$ that are just the ones that can be obtained with the gravitational boost discussed above.

The reason why the stau pair production is the dominant annihilation channel is twofold. The neutralino is bino-like thus the vertices's $\chi-\chi-Z$ and $\chi-\chi-(h, H)$ are suppressed, while the vertex $\chi-\tilde{\tau}_{1}-\tau$ is not suppressed by mixing. The dominant diagrams in Fig. 1 are thus the ones with $t$, $u$ channel exchange of the tau. Furthermore, at energies near the threshold the produced staus are slow thus the propagator $1 /\left(p_{\chi}^{2}-p_{\tilde{\tau}}\right)^{2}-m_{\tau}^{2}=1 /\left(m_{\tilde{\tau}}^{2}+m_{\chi}^{2}-2 E_{\chi} E_{\tilde{\tau}}+2 \mathbf{p}_{\tilde{\tau}} \cdot \mathbf{p}_{\chi}-m_{\tau}^{2}\right)$ is approximately $1 /\left[\left(m_{\tilde{\tau}}-m_{\chi}\right)^{2}-m_{\tau}^{2}\right]$. The cross section, proportional to the square of this quantity is, thus, enhanced for mass splittings approaching the tau mass.

\section{Gamma rays from non-monochromatic taus}

If the neutralino-stau mass splitting is larger than the tau mass, the staus can only decay into the two body final state $\chi \tau$, see diagrams in Fig. 1 . The tau pair will decay and radiate photons but with a peculiar spectrum. 

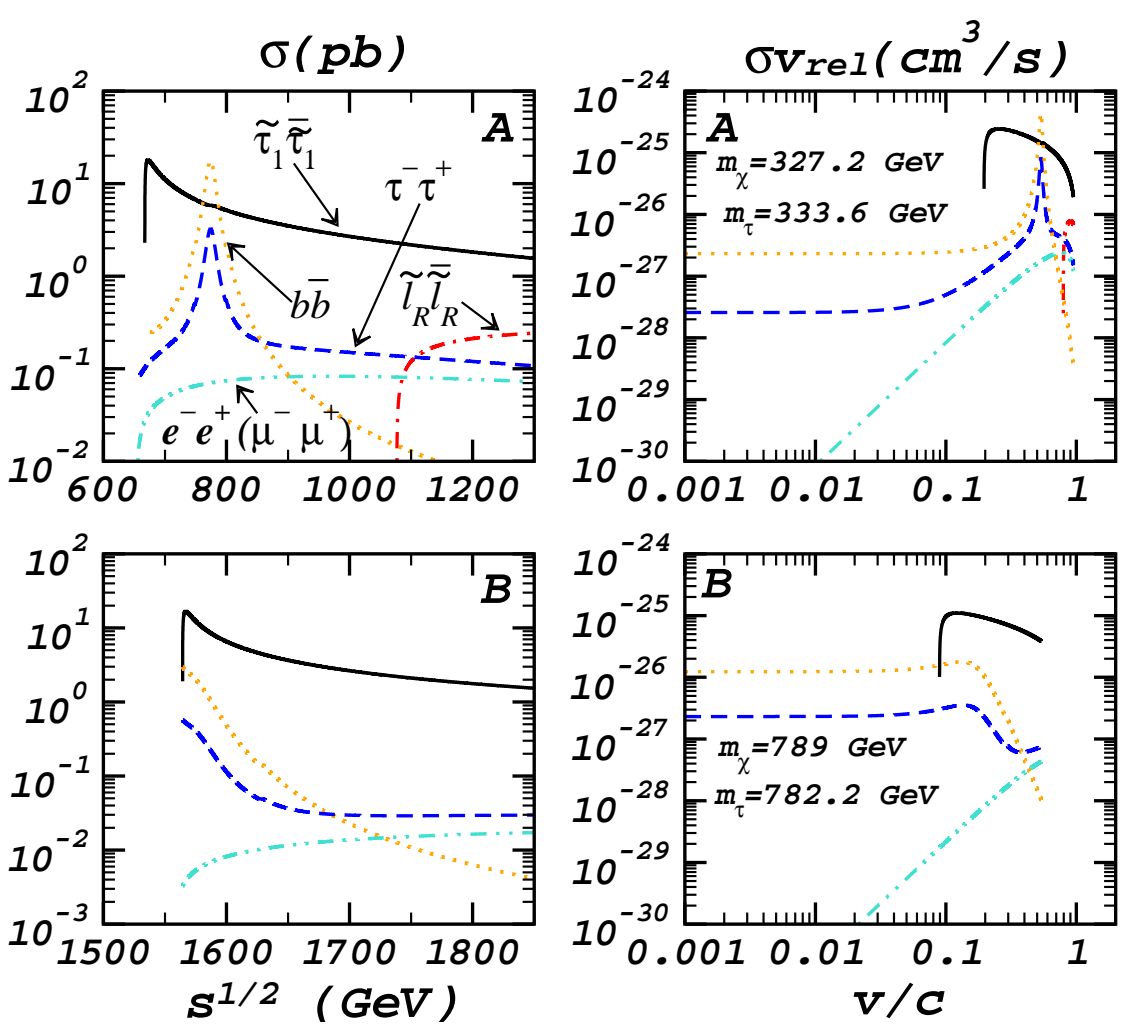

Figure 2: Annihilation cross sections in picobarn as a function of the CMF energy (left panels) and annihilation cross section times the relative velocity in $\mathrm{cm}^{3} / \mathrm{s}$ as a function of the colliding neutralino velocity (right panels). The CMSSM points A and B are specified in Ref. [5], the neutralino and stau masses are given.

The taus are not monochromatic and the spectrum changes with the collision energy $\sqrt{s}$ and ultimately with the distance, while in the static case the taus have an energy equal to the neutralino mass and the radiated photon spectrum is limited by $E_{\gamma}^{\max }=E_{\tau}=m_{\chi}$. The taus energy spectrum can be easily obtained by applying a Lorentz transformation with parameters $\beta=\left(1-4 m_{\tilde{\tau}}^{2} / s\right)^{1 / 2}$ and $\gamma=\sqrt{s} / 2 m_{\tilde{\tau}}$, to the spectrum calculated in the rest frame of the stau. In this frame it has fixed energy and momentum, $E_{\tau}^{*}=\left(m_{\tilde{\tau}}^{2}-m_{\chi}^{2}+m_{\tau}^{2}\right) / 2 m_{\tilde{\tau}}$ and $p_{\tau}^{*}=\left(E_{\tau}^{* 2}-m_{\tau}^{2}\right)^{1 / 2}$. The resulting energy distribution is flat and limited, $\Delta E=E_{\tau}^{\max }-E_{\tau}^{\min }$,

$$
\frac{d N\left(\tilde{\tau}_{1} \rightarrow \chi \tau\right)}{d E_{\tau}}=\frac{1}{\Delta E}, \quad E_{\tau}^{\min }=\gamma\left(E_{\tau}^{*}-\beta p_{\tau}^{*}\right) \leq E_{\tau} \leq E_{\tau}^{\max }=\gamma\left(E_{\tau}^{*}+\beta p_{\tau}^{*}\right) .
$$

Although the process is $2 \rightarrow 4$, applying the small width approximation to the stau propagators, and given that $B R\left(\tilde{\tau}_{1}^{ \pm} \rightarrow \tau^{ \pm} \chi\right)=1$, we have $\sigma\left(\chi \chi \rightarrow \tau^{-} \tau^{+} \chi \chi\right) \simeq \sigma\left(\chi \chi \rightarrow \tilde{\tau}_{1} \bar{\tau}_{1}\right) B R^{2}\left(\tilde{\tau}_{1} \rightarrow \tau \chi\right) \simeq$ $\sigma\left(\chi \chi \rightarrow \tilde{\tau}_{1} \bar{\tau}_{1}\right) \equiv \sigma_{\tilde{\tau} \tilde{\tau}}$. An example of the accuracy of these analytical approximations is shown in Fig. 3(a) where the histogram is obtained by calculating the energy distribution of the full $2 \rightarrow 4$ process with CalcHEP [15].

The number of photons with energy $E_{\gamma}$ produced by a tau with energy $E_{\tau}$ is given by $d N_{\gamma} / d x=$ $1 / 2 f(x)$ with $x=E_{\gamma} / E_{\tau}$ and $f(x)=x^{-3 / 2} \exp [g(x)]+q \log [p(1-x)]\left(x^{2}-2 x+2\right) / x$. This formula was obtained in Ref. [16], to which we refer the reader for details, by fitting the photon yield from 

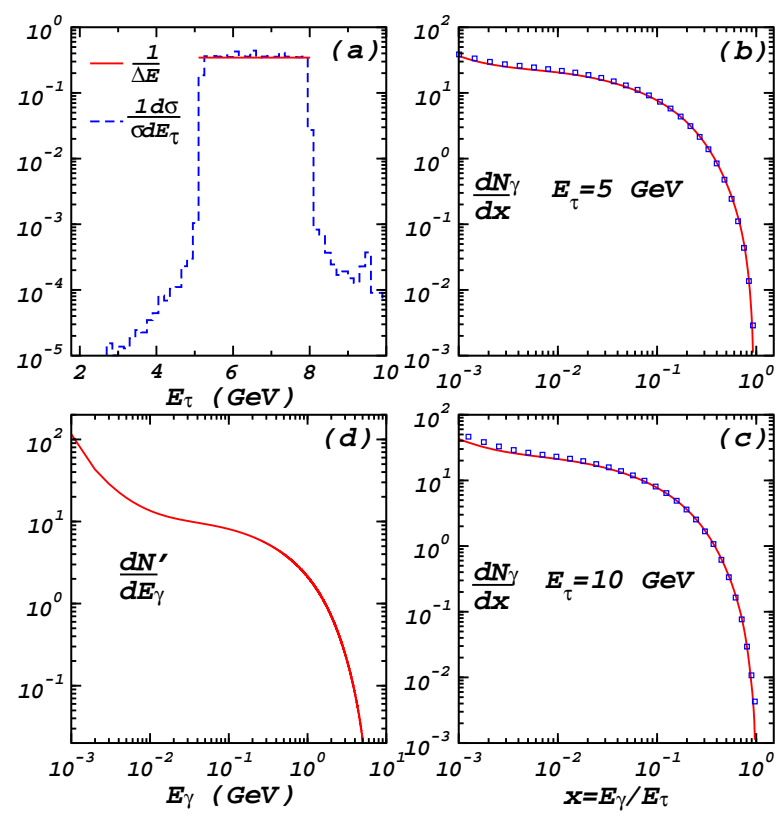

Figure 3: Panels (a) and (b) show the photon spectrum emitted by $\tau^{+} \tau^{-}$pairs with energy of the lepton fixed to 5 and $10 \mathrm{GeV}$. The points correspond to the simulated spectrum as given in the code MicrOMEGAs and the line is the the fit function discussed in the text. In panel (c) the red line is the tau spectrum, and the histogram is calculated with CalcHEP. Panel (d) shows the convoluted gamma-ray spectrum. In (c) and (d) we use the CMSSM point A and $\sqrt{s}=684 \mathrm{GeV}$.

taus obtained with Monte Carlo simulations of the non relativistic process $\chi \chi \rightarrow \tau^{+} \tau^{-}$. In this case the taus have equal energy, hence we use a factor $1 / 2$ for the yield of one particle. Although the fit is obtained for $m_{\mathrm{DM}}=E_{\tau} \geq 25 \mathrm{GeV}$, it works well also down to few $\mathrm{GeV}$ by confronting it with spectra as given in tables of MicrOMEGAs [17], see Fig. 3(b), Fig. 3(c).

The gamma spectrum at distance $r$ is then obtained by integrating over the tau energy distribution,

$$
\frac{d N}{d E_{\gamma}}(r)=\frac{1}{\Delta E(r)} \int_{E_{\tau}^{\min }(r)}^{E_{\tau}^{\max }(r)} \frac{d E_{\tau}}{E_{\tau}} f\left(\frac{E_{\gamma}}{E_{\tau}}\right) \theta\left(E_{\tau}-E_{\gamma}\right) .
$$

We have multiplied by 2 to obtain the yield of the pair. The Heaviside function takes into account that for fixed $E_{\gamma}$ the integrand is zero if $E_{\gamma}>E_{\tau}$. For this reason the photon energy cut-off is $E_{\tau}^{\min }$ for each $\sqrt{s}$, see Fig. 3(d) for an example.

\section{Flux}

The extension of the source is set by $r_{\max }$ that is too small to be resolved by present telescopes, thus we treat it as point source at the $\mathrm{GC}$ at a distance from us of $D=8 \mathrm{kpc}$. The differential photon flux as

$$
\frac{d \Phi}{d E_{\gamma}}=\frac{R_{\mathrm{S}}^{3}}{D^{2}} \int_{r_{\min }}^{r_{\max }} d r r^{2} \sigma_{\tilde{\tau} \tilde{\tau}}(r) v_{\mathrm{rel}}(r) \frac{\rho^{2}(r)}{m_{\chi}^{2}} \frac{d N}{d E_{\gamma}}(r) .
$$




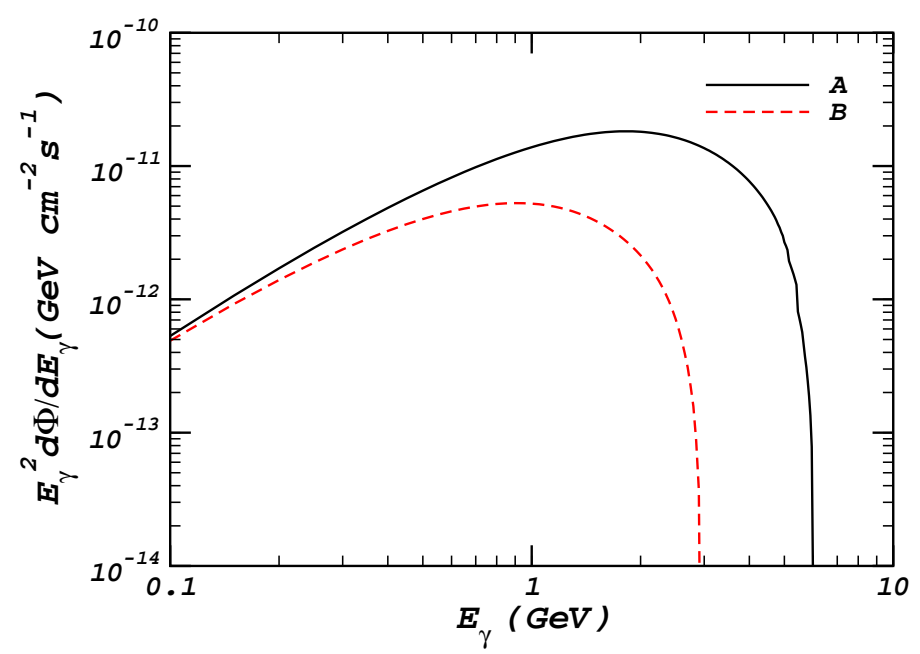

Figure 4: Differential flux for the two CMSSM reference points A and B.

In the integral distances are given in units of the Schwarzschild radius, thus $r$ is dimensionless and a factor $R_{\mathrm{S}}^{3}$ appears explicitly. The maximum radius is given by $r_{\max }=1 / 2\left[1-\left(m_{\tilde{\chi}} / m_{\tilde{\tau}}\right)^{2}\right] R_{\mathrm{S}}$. In contrast with the standard almost-static $\chi \chi \rightarrow \tau^{+} \tau^{-}$annihilation there is no factorization into a particle physics and an astrophysics factor because all the factors in the integrand depend on $r$ through the velocity dependence. Furthermore there is no factor $1 / 2$ in he flux because the final state necessarily contains two neutralinos, the number of dark matter particles is not changed by this process.

In Fig. 4 we show the differential flux. The absolute cut off when integrating over $\sqrt{s}$ is at $\gamma\left(r_{\max }\right) E_{\tau}^{*}$. In fact as $r \rightarrow r_{\max }, \beta \rightarrow 0$ and $E_{\tau}^{\min } \rightarrow \gamma\left(r_{\max }\right) E_{\tau}^{*}$. Note that $\gamma\left(r_{\max }\right) \sim 1$ and $E_{\tau}^{*} \lesssim$ $\left(m_{\tilde{\tau}}-m_{\chi}\right)$, thus the cut off is indicative of the mass splitting between the neutralino; the signal, then, shows up at energies below $10 \mathrm{GeV}$. It is expected that after 5 years operation, the Fermi-LAT satellite [18] reaches sensitivities of $10^{-10}-0.5 \times 10^{-11}$ photons $\mathrm{cm}^{-2} \mathrm{~s}^{-1}$ for energies between $0.5 \mathrm{GeV}$ and $10 \mathrm{GeV}$ and the signal can be one of the components observed by the collaboration.

\section{Acknowledgments}

It is a pleasure to thank Mario E. Gómez, Angeles Pérez-Garcia and J. D. Vergados for collaboration in Ref. [5] on which this presentation is based. This work is supported by a MultiDark under Grant No. CSD2009-00064 of the Spanish MICINN Consolider-Ingenio 2010 Program. Further support is provided by the MICINN project FPA2011-23781 and from the Grant MICINNINFN(PG21)AIC-D-2011-0724.

\section{References}

[1] J. R. Ellis, T. Falk, K. A. Olive and M. Srednicki, Calculations of neutralino-stau coannihilation channels and the cosmologically relevant region of MSSM parameter space, Astropart. Phys. 13, 181 (2000), [Erratum-ibid. 15, 413 (2001)]; [hep-ph/9905481]. M. E. Gomez, G. Lazarides and C. Pallis, 
Supersymmetric cold dark matter with Yukawa unification, Phys. Rev. D 61, 123512 (2000) [hep-ph/9907261].

[2] G. Aad et al. [ATLAS Collaboration], Observation of a new particle in the search for the Standard Model Higgs boson with the ATLAS detector at the LHC, Phys. Lett. B 716, 1 (2012) [1207.7214]; S. Chatrchyan et al. [CMS Collaboration], Observation of a new boson at a mass of $125 \mathrm{GeV}$ with the CMS experiment at the LHC, Phys. Lett. B 716, 30 (2012) [1207.7235].

[3] G. F. Giudice, P. Paradisi and A. Strumia Correlation between the Higgs Decay Rate to Two Photons and the Muon $g$ - 2, JHEP 1210 (2012) 186 [1207.6393].

[4] M. Banados, J. Silk and S. M. West, Kerr Black Holes as Particle Accelerators to Arbitrarily High Energy, Phys. Rev. Lett. 103, 111102 (2009) [0909.0169].

[5] M. Cannoni, M. E. Gomez, M. A. Perez-Garcia and J. D. Vergados, New gamma ray signal from gravitationally boosted neutralinos at the galactic center, Phys. Rev. D 85, 115015 (2012) [1205.1709].

[6] M. A. Amin and T. Wizansky, Relativistic dark matter at the Galactic center, Phys. Rev. D 77, 123510 (2008) [0710.5517].

[7] P. Gondolo and J. Silk, Dark matter annihilation at the galactic center, Phys. Rev. Lett. 83, 1719 (1999) [astro-ph/9906391].

[8] D. Merritt, Dark matter at the centres of galaxies, in Particle Dark Matter: Observations, Models and Searches, edited by G. Bertone, Cambridge University Press (2010) [1001.3706].

[9] O. Y. Gnedin and J. R. Primack, Dark Matter Profile in the Galactic Center, Phys. Rev. Lett. 93, 061302 (2004) [astro-ph/0308385].

[10] D. Merritt, Evolution of the dark matter distribution at the galactic center, Phys. Rev. Lett. 92, 201304 (2004) [astro-ph/0311594].

[11] G. Bertone and D. Merritt, Dark matter dynamics and indirect detection, Mod. Phys. Lett. A 20, 1021 (2005) [astro-ph/0504422].

[12] E. Vasiliev, Dark matter annihilation near a black hole: Plateau vs. weak cusp, Phys. Rev. D 76, 103532 (2007) [0707.3334]; E. Vasiliev and M. Zelnikov, Dark matter dynamics in Galactic center, Phys. Rev. D 78, 083506 (2008) [0803.0002].

[13] G. R. Blumenthal, S. M. Faber, R. Flores and J. R. Primack, Contraction of Dark Matter Galactic Halos Due to Baryonic Infall, Astrophys. J. 301, 27 (1986); F. Prada, A. Klypin, J. Flix Molina, M. Martinez and E. Simonneau, Dark Matter Annihilation in the Milky Way Galaxy: Effects of Baryonic Compression, Phys. Rev. Lett. 93, 241301 (2004) [astro-ph/0401512]. Y. Mambrini, C. Munoz, E. Nezri and F. Prada, Adiabatic compression and indirect detection of supersymmetric dark matter, J. Cosmol. Astropart. Phys. 01 (2006) 010 [hep-ph/0506204].

[14] J. Binney and S. Tremaine, Galactic Dynamics, Princeton University Press, Princeton, NJ, 1987.

[15] A. Pukhov, A. Belyaev and N. Christensen, http://theory.sinp.msu.ru/ pukhov/calchep.html.

[16] J. A. R. Cembranos, A. de la Cruz-Dombriz, A. Dobado, R. A. Lineros and A. L. Maroto, Photon spectra from WIMP annihilation, Phys. Rev. D 83, 083507 (2011) [1009.4936].

[17] G. Belanger et al. , Indirect search for dark matter with micrOMEGAs2.4, Comput. Phys. Commun. 182, 842 (2011) [1004.1092].

[18] V. Vitale et al. [Fermi-LAT Collaboration], Search for dark matter with Fermi Large Area Telescope: The galactic center, Nucl. Instrum. Meth. A 630, 147 (2011). 